\title{
Essential thrombocythemia treatment algorithm 2018
}

\author{
Ayalew Tefferi ${ }^{1}$, Alessandro M. Vannucchi ${ }^{2}$ and Tiziano Barbui ${ }^{3}$
}

\begin{abstract}
Current drug therapy for myeloproliferative neoplasms, including essential thrombocythemia (ET) and polycythemia vera (PV), is neither curative nor has it been shown to prolong survival. Fortunately, prognosis in ET and PV is relatively good, with median survivals in younger patients estimated at 33 and 24 years, respectively. Therefore, when it comes to treatment in $\mathrm{ET}$ or $\mathrm{PV}$, less is more and one should avoid exposing patients to new drugs that have not been shown to be disease-modifying, and whose long-term consequences are suspect (e.g., ruxolitinib). Furthermore, the main indication for treatment in ET and PV is to prevent thrombosis and, in that regard, none of the newer drugs have been shown to be superior to the time-tested older drugs (e.g., hydroxyurea). We currently consider three major risk factors for thrombosis (history of thrombosis, JAK2/MPL mutations, and advanced age), in order to group ET patients into four risk categories: "very low risk" (absence of all three risk factors); "low risk" (presence of JAK2/MPL mutations); "intermediate-risk" (presence of advanced age); and "high-risk" (presence of thrombosis history or presence of both JAK2/MPL mutations and advanced age). Herein, we provide a point-of-care treatment algorithm that is risk-adapted and based on evidence and decades of experience.
\end{abstract}

\section{Introduction}

The term myeloproliferative neoplasms (MPN) typically refers to essential thrombocythemia (ET), polycythemia vera $(\mathrm{PV})$, and primary myelofibrosis $(\mathrm{PMF})^{1}$; in addition, some patients with ET or PV might in time progress into a PMF-like post-ET or post-PV myelofibrosis ${ }^{2}$. As a group, $\mathrm{ET}, \mathrm{PV}$, and PMF share three mutually exclusive "driver" mutations, including JAK2, CALR, and $M P L^{3}$. The most frequent driver mutation is $J A K 2 \mathrm{~V} 617 \mathrm{~F}$, found in $~ 99 \%$ of patients with $\mathrm{PV}, 55 \% \mathrm{ET}$, and $65 \% \mathrm{PMF}^{4}$. The driver mutation distributions in ET and PMF are similar with $50-65 \%$ of the patients being JAK2 V617F mutated, $15-30 \%$ being CALR mutated, and $4-8 \%$ being $M P L$ mutated $^{4}$, while $10-20 \%$ of the patients might not express any one of the three mutations (i.e., are triple-negative) ${ }^{4}$.

Correspondence: Ayalew Tefferi (tefferi.ayalew@mayo.edu)

${ }^{1}$ Division of Hematology, Department of Medicine, Mayo Clinic, Rochester, MN, USA

2Department of Experimental and Clinical Medicine, CRIMM, Center Research and Innovation of Myeloproliferative Neoplasms, Azienda Ospedaliera

Universitaria Careggi, University of Florence, Florence, Italy

Full list of author information is available at the end of the article
World Health Organization (WHO)-consistent diagnosis of ET requires a platelet count of $\geq 450 \times 10(9) / \mathrm{L}$, presence of one of the three aforementioned driver mutations or in their absence the exclusion of other causes of thrombocytosis (reactive and clonal), and bone marrow morphologic assessment, especially for distinguishing ET from prefibrotic PMF and "masked" PV $V^{5,6}$. In addition to clonal thrombocytosis, a variable proportion of patients with ET might display mild splenomegaly, leukocytosis, microvascular symptoms, thrombotic and bleeding complications, increased occurrence of first trimester miscarriage, and time-dependent risk of leukemic transformation or fibrotic progression ${ }^{7}$.

Survival in patients with any one of the three JAK2 mutation-enriched MPN is significantly shorter than that of the sex- and age-adjusted control population, with median estimates of 20 years for ET, 14 years for PV, and 6 years for $\mathrm{PMF}^{8}$. Causes of death include leukemic transformation, with 15 -year estimates of $\sim 2.1-5.3 \%$ for ET, $5.5-18.7 \%$ for $\mathrm{PV}$, and more than $20 \%$ for $\mathrm{PMF}^{9}$. Fibrotic progression rates in ET and PV, during a similar 
time interval, are estimated at $4-11 \%$ and $6-14 \%$, respectively ${ }^{9}$. To date, drug therapy has not been shown to modify the natural history of these diseases, prevent leukemic or fibrotic progression or prolong survival ${ }^{10}$. Current indication for drug therapy in ET and PV is to prevent thrombotic complications, especially in high-risk patients ${ }^{7}$. In the current review, we provide a risk-adapted treatment algorithm in ET that can be used in daily practice.

\section{Risk-adapted treatment algorithm in essential thrombocythemia}

Survival and its prognostic determinants

Life expectancy in ET is only mildly compromised with median survival for patients younger than 60 years of age approaching 33 years $^{8}$. In addition to age, other clinical risk factors for survival in ET include leukocytosis and thrombosis history ${ }^{11}$. On the other hand, neither abnormal karyotype (detected in $\sim 7 \%$ of patients) ${ }^{12}$ nor driver mutational status ${ }^{13}$ in ET has been shown to affect overall or leukemia-free survival; however, JAK2/MPL-mutated patients are significantly more thrombosis prone while MPL-mutated cases might be at a higher risk for fibrotic progression $^{13-15}$.

A recent targeted sequencing study revealed that mutations or DNA variants, other than JAK2, CALR, or $M P L$, are found in $\sim 53 \%$ of patients with ET with the most frequent being TET2 (16\%), ASXL1 (11\%), DNMT3A (6\%), and $S F 3 B 1(5 \%)^{16}$. The particular study identified SH2B3, SF3B1, U2AF1, TP53, IDH2, and EZH2 mutations as risk factors for overall, myelofibrosis-free or leukemiafree survival; at least one of these mutations was seen in $\sim 15 \%$ of the patients and median survival of patients with and without adverse mutations were 9 and 22 years, respectively. Furthermore, the effect on survival from these adverse mutations was not accounted for by current clinically devised prognostic models and the observations were validated in an external cohort of patients ${ }^{16}$. Most recently, serum lactate dehydrogenase (LDH) level in ET was shown to correlate with shortened survival, suggesting its value as a biologically more accurate measure of myeloproliferation (as opposed to leukocytosis) and possible surrogate for occult prefibrotic $\mathrm{PMF}^{17}$.

Taking the above discussion into consideration, it is important to identify the risk factor-free subset of ET patients, since their survival might not be significantly different from the age- and sex-matched control population; such patients are represented by morphologic confirmation of WHO-defined ET (as opposed to prefibrotic PMF), younger age, absence of thrombosis history, absence of leukocytosis, normal LDH, and absence of $M P L$ or other adverse mutations, as outlined above. On the other hand, the presence of risk factors for survival is currently not used to dictate treatment, since specific therapy in ET has not been shown to affect survival. Accordingly, although advised after securing insurance coverage and patient permission, we do not believe it is currently crucial to obtain next-generation sequencing (NGS) in ET. In other words, at the present time, identification of survival risk factors in ET is used to counsel patients and disease-monitoring purposes and not for treatment decisions.

\section{Thrombosis and its prognostic determinants}

Current treatment in ET is primarily indicated for the purposes of preventing thrombotic complications, which might occur in $10-20 \%$ of patients. In this regard, the twotiered traditional risk stratification considers two risk parameters: age $>60$ years and history of thrombosis. Accordingly, patients with either one of these two risk factors were classified as "traditional high-risk" and the absence of both risk factors defined the "traditional lowrisk" groups. More recently, however, several studies have identified the presence of JAK2/MPL mutations as another independent risk factor for thrombosis in $\mathrm{ET}^{18,19}$. More specifically, risk factors for arterial thrombosis included thrombosis history, age $>60$ years, presence of $J A K 2 \mathrm{~V} 617 \mathrm{~F}$, leukocytosis, and CV risk factors and for venous thrombosis male gender ${ }^{19}$, while a lower risk of thrombosis was shown in patients with extreme thrombocytosis $^{19}$ and in those with CALR mutations ${ }^{20,21}$.

\section{Contemporary risk stratification}

Thrombosis data from 1019 patients with WHOdefined ET was recently re-analyzed ${ }^{18}$; among the "traditional low-risk" group, annual thrombosis rate was the lowest in patients who lacked both JAK2/MPL mutations and CV risk factors (0.44\%), non-significantly higher in JAK2-unmutated patients with CV risk factors $(1.05 \%)$ and significantly higher in $J A K 2$-mutated patients with (2.57\%) or without (1.59\%) CV risk factors; there was no significant difference between JAK2-mutated "traditional low-risk" patients with or without CV risk factors. In the "traditional high-risk" group, the particular study ${ }^{18}$ identified thrombosis history as being significantly more detrimental than advanced age and also showed that the adverse effect of $J A K 2$ mutations was more apparent in patients whose high-risk status was determined by advanced age while its additional effect on patients with thrombosis history was limited; these observations from the revised international prognostic scoring system for essential thrombocythemia thrombosis were recently validated by another study ${ }^{22}$.

Based on the above, we currently consider four risk groups in ET: "very low-risk group" is defined by the absence of all three independent risk factors for thrombosis, including history of thrombosis, JAK2/MPL mutations, and advanced age; "low-risk" group is defined by the 


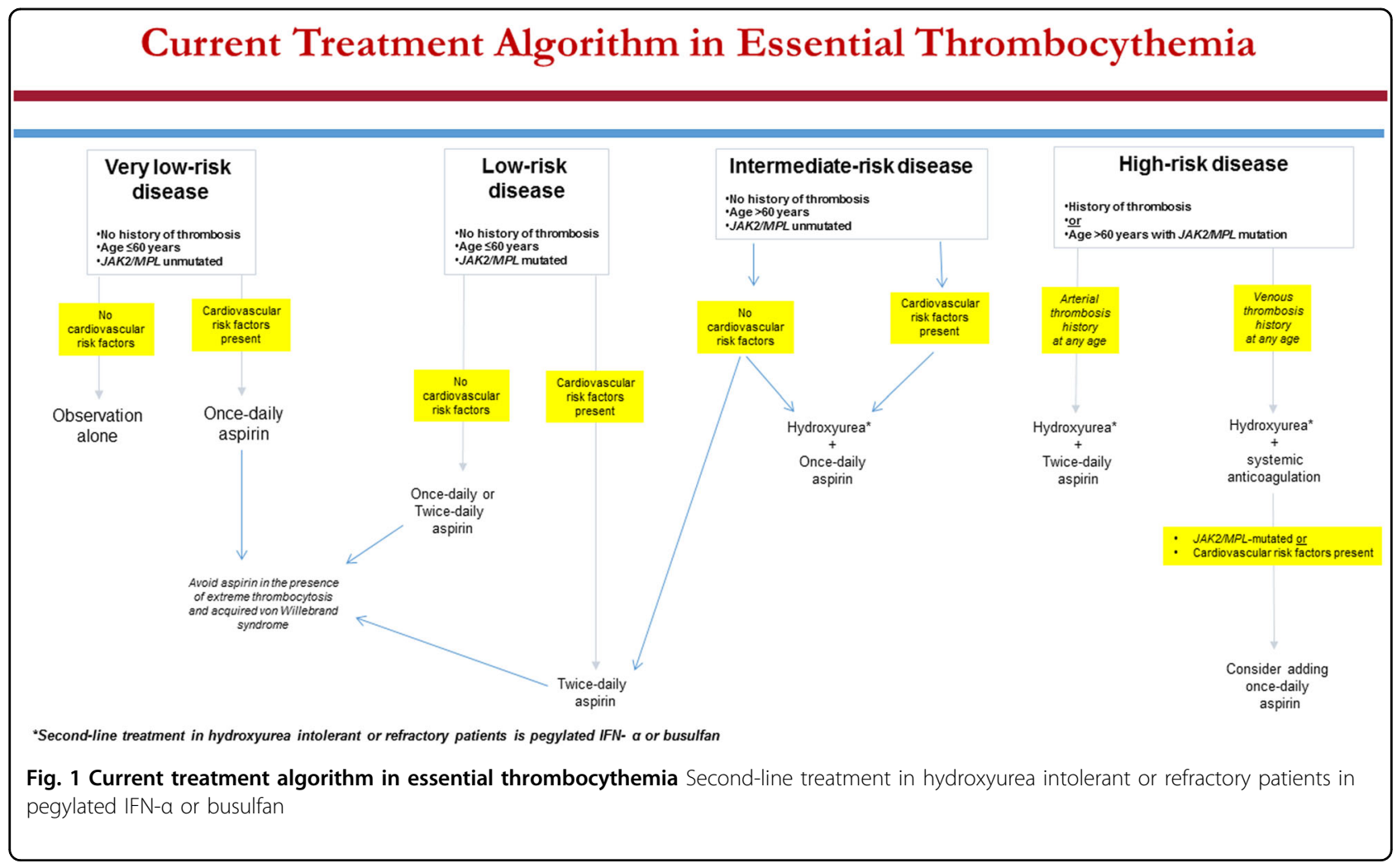

presence of $J A K 2 / M P L$ mutations in otherwise younger patients without history of thrombosis; "intermediaterisk" group refers to $J A K 2 / M P L$ unmutated older patients without thrombosis history; and "high-risk" group is defined by either presence of thrombosis history or presence of $J A K 2 / M P L$ mutation in an older patient (Fig. 1).

\section{Risk-adapted therapy: "very low-risk" disease}

At present, there is no evidence from prospective controlled studies to guide treatment recommendations for each one of the above-mentioned four risk categories in ET. Until such information becomes available, it is reasonable to simply observe patients with "very low-risk" disease without $\mathrm{CV}$ risk factors and consider once-daily aspirin therapy only in the presence of CV risk factors (Fig. 1). In other words, aspirin therapy in "very low-risk" ET should not be automatic, especially considering the fact that a substantial proportion of such patients display acquired von Willebrand syndrome (AVWS) with increased bleeding diathesis ${ }^{23}$; this particular complication is more likely to occur in the presence of extreme thrombocytosis ${ }^{24}$. Furthermore, other studies have suggested the value of aspirin therapy in reducing the risk of arterial thrombosis in low-risk ET associated with CV risk factors, but not otherwise ${ }^{25}$. Because patients with "very low-risk" ET are either CALR mutated or triple-negative, they usually display extreme thrombocytosis, which does not require specific treatment per se, regardless of how high the platelet count might be, as long as patient remains asymptomatic. On the other hand, if such patients with extreme thrombocytosis develop symptoms or bleeding complications, it is reasonable to use a cytoreductive agent, with a goal of keeping the platelet count commensurate with the resolution of the particular symptom.

\section{Risk-adapted therapy: "low-risk" disease}

In "low-risk" ET (i.e., young JAK2/MPL-mutated without thrombosis history), the aforementioned recent studies have disclosed a residual risk of thrombosis despite management according to traditional treatment guidelines ${ }^{18,26}$. Therefore, it is reasonable to consider further optimization of aspirin therapy in such patients by following "twice-daily" rather than "once-daily" schedule, especially in the presence of $\mathrm{CV}$ risk factors ${ }^{26}$. The rationale for twice-daily aspirin dosing in "low-risk" JAK2/ $M P L$-mutated ET patients is primarily based on emerging data on the inadequacy of once-daily aspirin dosing for 24-h optimal suppression of thromboxane-A2 synthesis, in the presence of high platelet turnover, and demonstration of superior biological efficacy in ET with twicedaily dosing ${ }^{27,28}$.

\section{Risk-adapted therapy: "intermediate-risk" disease}

Recent studies have suggested that "advanced age," by itself, was a weak risk factor for thrombosis and may not 
be as detrimental as thrombosis history ${ }^{18,26}$. These observations have led us to split the "traditionally highrisk" ET category into "intermediate risk," defined by the presence of advanced age without history of thrombosis or JAK2/MPL mutations, and "high risk," defined by presence of thrombosis history or presence of both advanced age and $J A K 2 / M P L$ mutations. Such distinction is therapeutically relevant since it provides the option of avoiding cytoreductive therapy in $J A K 2 / M P L$ unmutated older patients without history of thrombosis or $\mathrm{CV}$ risk factors (Fig. 1); in one of the aforementioned studies ${ }^{18}$, the annual risk of thrombosis in such patients was $1.44 \%$, compared to $4.17 \%$ in the presence of both JAK2 mutations and CV risk factors $(p=0.01)$, and was similar to that of "low-risk" patients (1.59-2.57\%). Accordingly, we do not believe that it is mandatory to use cytoreductive therapy in such patients (Fig. 1).

\section{Risk-adapted therapy: "high-risk" disease}

Decades ago, "high-risk" disease in ET was defined by the presence of one of three clinical parameters: history of thrombosis, advanced age, and long duration of thrombocytosis $^{29}$. Subsequently, in a randomized study using hydroxyurea for high-risk disease, patients with platelet count $>1500 \times 10(9) / \mathrm{L}$ were excluded because it was felt that such patients required treatment because of increased bleeding diathesis ${ }^{30}$. Over the years, it has become evident that extreme thrombocytosis in ET did not, by itself, increase thrombosis risk and might actually be associated with a reduced risk of arterial thrombosis $^{19,31}$. Also, the bleeding diathesis associated with extreme thrombocytosis has been linked to AVWS ${ }^{24}$, which might occur both in the presence and absence of extreme thrombocytosis ${ }^{23}$, and is effectively screened for and managed appropriately. Therefore, platelet count per se should no longer be used for risk stratification in ET.

Regardless, management of "traditionally high-risk" ET has been primarily guided by results of a randomized study of hydroxyurea vs no cytoreductive treatment, in high-risk patients, with the goal of keeping the platelet count below $600 \times 10(9) / \mathrm{L}^{30}$; the study showed a statistically significant benefit for hydroxyurea therapy (thrombosis rate of 3.6 vs 24\%). Since then, unsuccessful attempts have been made to improve upon hydroxyurea treatment in $\mathrm{ET}^{32,33}$. Accordingly, hydroxyurea, combined with once-daily aspirin therapy ${ }^{34}$, remains the standard of care for contemporarily classified "high-risk" patients (Fig. 1). However, there is room for improvement in our conventional treatment approach ${ }^{18,26}$ and we underscore the need to maximize anti-thrombotic activity, by shortening the aspirin dosing schedule to every $12 \mathrm{~h}$, for patients with history of arterial thrombosis, and securing long-term systemic anticoagulation, in patients with history of venous thrombosis (Fig. 1). In addition, it is reasonable to continue with once-daily aspirin therapy, along with systemic anticoagulation, in patients who are at risk for arterial thrombosis (Fig. 1). In this regard, there is evidence for the additional value of aspirin therapy in the prevention of recurrent venous thrombosis ${ }^{35,36}$.

\section{Treatment options for hydroxyurea intolerant or refractory patients}

There are currently four drugs to consider as secondline therapy in ET: pegylated interferon- $\alpha$ (IFN- $\alpha$ ), busulfan, anagrelide, and pipobroman. Among these, our current choice for second-line therapy is pegylated IFN- $\alpha$ (starting dose $90 \mathrm{mcg}$ SC weekly). Pegylated IFN- $\alpha$ treatment in ET has been shown to be relatively safe and effective, and has been associated with both clinical (70-80\%) and molecular (10-20\%) remissions in some patients, especially in the presence of CALR mutations ${ }^{37,38}$; however, the relevance of the latter observation, in terms of meaningful health outcome, remains uncertain. Busulfan (starting dose $2-4 \mathrm{mg}$ /day) is a reasonable alternative drug for second-line therapy in ET and it too has been shown to be safe and effective as well as induce molecular remissions in both ET and PV ${ }^{39,40}$; in hydroxyurea intolerant or refractory patients with ET or PV, the drug was shown to induce durable hematologic response in the majority of patients and molecular response in a minority ${ }^{41-43}$. In addressing the ongoing concern regarding drug leukemogenicity, a large international study of over 1500 patients with PV found no evidence that implicated busulfan, IFN- $\alpha$, or hydroxyurea, while confirming the particular association with pipobroman ${ }^{44}$. In a noteworthy vote of confidence regarding busulfan use in MPN, a prominent hematologist underscored the fact that busulfan displayed less DNA/RNA binding, compared to other alkylating agents, no inter- or intra-strand DNA binding and no immunosuppression ${ }^{45}$.

Anagrelide has been evaluated, in controlled studies, for its efficacy and safety as first-line therapy for $\mathrm{ET}^{32,33}$; the results of these studies suggested that anagrelide was not inferior to hydroxyurea in one study ${ }^{33}$, but might have been harmful to patients in the second study ${ }^{32}$. In the latter study, patients receiving anagrelide experienced higher incidences of arterial thrombosis, bleeding complications, and fibrotic progression. Similarly, noncontrolled studies have suggested that more than a quarter of patients receiving anagrelide therapy become anemic while a lesser percentage experience renal insufficiency and cardiac complications including arrhythmia and cardiomyopathy ${ }^{46-50}$. Therefore, we currently consider anagrelide therapy only after failure of all other drug options, including hydroxyurea, IFN- $\alpha$, and busulfan. Finally, despite some uncontrolled reports of safety and efficacy $^{51-53}$, we currently do not recommend 
pipobroman treatment in ET, because of controlled evidence for leukemogenicity, seen in patients with $\mathrm{PV}^{54}$.

\section{Management during pregnancy}

Current treatment recommendations in young women wishing to be pregnant or are pregnant include once-daily aspirin for "very low-risk" or "low-risk" disease and pegylated IFN- $\alpha$ for high-risk disease ${ }^{55}$. Both aspirin and IFN- $\alpha$ therapy have been shown to be safe for use during pregnancy and might be associated with lower miscarriage rates in women with $\mathrm{ET}^{55-57}$. The additional value of other measures, including platelet apheresis or low molecular weight heparin, is unclear and not recommended ${ }^{58}$.

\section{Conclusions}

The most important first step in the management of ET is to confirm the accuracy of the diagnosis and make sure that other myeloid neoplasms, which might mimic ET in their presentation (e.g., prefibrotic PMF, masked polycythemia vera, chronic myeloid leukemia, refractory anemia with ring sideroblasts, and thrombocytosis), are excluded. Most patients with WHO-defined ET can expect a normal life expectancy with very low risk of leukemic transformation or fibrotic progression and a diagnosis of ET should not deter one from continuing with normal life activities, including sports, air travel, and pregnancy. Patients with ET should be informed about their driver mutational status and its prognostic and therapeutic implications. In this regard, aspirin therapy is very important for JAK2-mutated patients, because of their increased risk for arterial thrombosis. MPL mutations are infrequent in ET $(\sim 3 \%)$ and their presence raises the possibility of occult prefibrotic PMF or an increased risk of fibrotic progression. Observation alone remains a viable treatment option for "very low-risk" patients with ET while all other patients might benefit from aspirin therapy, in a once- or twice-daily schedule. In addition, cytoreductive treatment is strongly encouraged in patients with thrombosis history, and our first- and second-line drugs of choice in this regard are hydroxyurea and pegylated IFN- $\alpha$, respectively. On the other hand, we no longer insist on the use of cytoreductive therapy in older patients without previous vascular events, provided they are $J A K 2 / M P L$ unmutated.

\section{Author details}

'Division of Hematology, Department of Medicine, Mayo Clinic, Rochester, MN, USA. ${ }^{2}$ Department of Experimental and Clinical Medicine, CRIMM, Center Research and Innovation of Myeloproliferative Neoplasms, Azienda Ospedaliera Universitaria Careggi, University of Florence, Florence, Italy. ${ }^{3}$ Research Foundation Papa Giovanni XXIII Hospital Bergamo, Italy

\section{Conflict of interest}

The authors declare that they have no conflict of interest.

\section{Publisher's note}

Springer Nature remains neutral with regard to jurisdictional claims in published maps and institutional affiliations.

Received: 10 July 2017 Accepted: 20 July 2017

Published online: 10 January 2018

\section{References}

1. Arber, D. A. et al. The 2016 revision to the World Health Organization classification of myeloid neoplasms and acute leukemia. Blood 127, 2391-2405 (2016).

2. Barosi, G. et al. Proposed criteria for the diagnosis of post-polycythemia vera and post-essential thrombocythemia myelofibrosis: a consensus statement from the International Working Group for Myelofibrosis Research and Treatment. Leukemia 22, 437-438 (2008).

3. Tefferi, A. Myeloproliferative neoplasms: a decade of discoveries and treatment advances. Am. J. Hematol. 91, 50-58 (2016).

4. Tefferi, A. \& Pardanani, A. Myeloproliferative neoplasms: a contemporary review. JAMA Oncol. 1, 97-105 (2015).

5. Barbui, T. et al. The 2016 revision of WHO classification of myeloproliferative neoplasms: clinical and molecular advances. Blood Rev. 30, 453-459 (2016).

6. Kvasnicka H. M. et al. European LeukemiaNet study on the reproducibility of bone marrow features in masked polycythemia vera and differentiation from essential thrombocythemia. Am. J. Hematol. 92, 1062-1067 (2017).

7. Tefferi, A. \& Barbui, T. Polycythemia vera and essential thrombocythemia: 2017 update on diagnosis, risk-stratification, and management. Am. J. Hematol. 92, 94-108 (2017)

8. Tefferi, A. et al. Long-term survival and blast transformation in molecularly annotated essential thrombocythemia, polycythemia vera, and myelofibrosis. Blood 124, 2507-2513 (2014). quiz 2615.

9. Cerquozzi, S. \& Tefferi, A. Blast transformation and fibrotic progression in polycythemia vera and essential thrombocythemia: a literature review of incidence and risk factors. Blood Cancer J. 5, e366 (2015).

10. Cervantes, F. \& Pereira, A. Does ruxolitinib prolong the survival of patients with myelofibrosis? Blood 129, 832-837 (2017).

11. Passamonti, F. et al. A prognostic model to predict survival in 867 World Health Organization-defined essential thrombocythemia at diagnosis: a study by the International Working Group on Myelofibrosis Research and Treatment. Blood 120, 1197-1201 (2012).

12. Gangat, N. et al. Cytogenetic abnormalities in essential thrombocythemia: prevalence and prognostic significance. Eur. J. Haematol. 83, 17-21 (2009).

13. Elala, Y. C. et al. Calreticulin variant stratified driver mutational status and prognosis in essential thrombocythemia. Am. J. Hematol. 91, 503-506 (2016).

14. Haider, M., Elala, Y. C., Gangat, N., Hanson, C. A. \& Tefferi, A. MPL mutations and palpable splenomegaly are independent risk factors for fibrotic progression in essential thrombocythemia. Blood Cancer J. 6, e487 (2016).

15. Tefferi, A. et al. Calreticulin mutations and long-term survival in essential thrombocythemia. Leukemia 28, 2300-2303 (2014).

16. Tefferi, A. et al. Targeted deep sequencing in polycythemia vera and essential thrombocythemia. Blood Adv. 1, 21-30 (2016).

17. Mudireddy, M. et al. The prognostic relevance of serum lactate dehydrogenase and mild bone marrow reticulin fibrosis in essential thrombocythemia. Am. J. Hematol. 92, 454-459 (2017).

18. Barbui, T. et al. Practice-relevant revision of IPSET-thrombosis based on 1019 patients with WHO-defined essential thrombocythemia. Blood Cancer J. 5, e369 (2015)

19. Carobbio, A. et al. Risk factors for arterial and venous thrombosis in WHOdefined essential thrombocythemia: an international study of 891 patients. Blood 117, 5857-5859 (2011).

20. Finazzi, G. et al. Calreticulin mutation does not modify the IPSET score for predicting the risk of thrombosis among 1150 patients with essential thrombocythemia. Blood 124, 2611-2612 (2014).

21. Rotunno, G. et al. Impact of calreticulin mutations on clinical and hematological phenotype and outcome in essential thrombocythemia. Blood 123, 1552-1555 (2014)

22. Haider, M. et al. Validation of the revised International Prognostic Score of Thrombosis for Essential Thrombocythemia (IPSET-thrombosis) in 585 Mayo Clinic patients. Am. J. Hematol. 91, 390-394 (2016). 
23. Rottenstreich, A. et al. Factors related to the development of acquired von Willebrand syndrome in patients with essential thrombocythemia and polycythemia vera. Eur. J. Intern. Med. 41, 49-54 (2017).

24. Michiels, J. J. Acquired von Willebrand disease due to increasing platelet count can readily explain the paradox of thrombosis and bleeding in thrombocythemia. Clin. Appl. Thromb. Hemost. 5, 147-151 (1999).

25. Alvarez-Larran, $\mathbf{A}$. et al Observation versus antiplatelet therapy as primary prophylaxis for thrombosis in low-risk essential thrombocythemia. Blood $\mathbf{1 1 6}$ 1205-1210 (2010).

26. Barbui, $\mathrm{T}$. et al Development and validation of an International Prognostic Score of thrombosis in World Health Organization-essential thrombocythemia (IPSET-thrombosis). Blood 120, 5128-5133 (2012).

27. Dillinger, J. G. et al. Twice daily aspirin to improve biological aspirin efficacy in patients with essential thrombocythemia. Thromb. Res. 129, 91-94 (2012)

28. Pascale, S. et al. Aspirin-insensitive thromboxane biosynthesis in essentia thrombocythemia is explained by accelerated renewal of the drug target. Blood 119, 3595-3603 (2012)

29. Cortelazzo, S. et al. Incidence and risk factors for thrombotic complications in a historical cohort of 100 patients with essential thrombocythemia. J. Clin. Oncol. 8, 556-562 (1990).

30. Cortelazzo, S. et al. Hydroxyurea for patients with essential thrombocythemia and a high risk of thrombosis. N. Engl. J. Med. 332, 1132-1136 (1995).

31. Latagliata, R. et al. High platelet count at diagnosis is a protective factor for thrombosis in patients with essential thrombocythemia. Thromb. Res. 156, 168-171 (2017)

32. Harrison, C. N. et al. Hydroxyurea compared with anagrelide in high-risk essential thrombocythemia. N. Engl. J. Med. 353, 33-45 (2005)

33. Gisslinger, $\mathrm{H}$. et al. Anagrelide compared with hydroxyurea in WHO-classified essential thrombocythemia: the ANAHYDRET Study, a randomized controlled trial. Blood 121, 1720-1728 (2013).

34. Alvarez-Larran, A. et al. Cytoreduction plus low-dose aspirin versus cytoreduction alone as primary prophylaxis of thrombosis in patients with high-risk essential thrombocythaemia: an obsenvational study. Br. J. Haematol. 161, 865-871 (2013).

35. Becattini, $C$. et al. Aspirin for preventing the recurrence of venous thromboembolism. N. Engl. J. Med. 366, 1959-1967 (2012)

36. Antiplatelet Trialists' Collaboration. Collaborative overview of randomised trials of antiplatelet therapy--III: reduction in venous thrombosis and pulmonary embolism by antiplatelet prophylaxis among surgical and medical patients. BMJ. 308, 235-246 (1994).

37. Verger, E. et al. Clinical and molecular response to interferon-alpha therapy in essential thrombocythemia patients with CALR mutations. Blood 126 2585-2591 (2015)

38. Quintas-Cardama, A. et al. Molecular analysis of patients with polycythemia vera or essential thrombocythemia receiving pegylated interferon alpha-2a. Blood 122, 893-901 (2013).

39. Shvidel, L. et al. Busulphan is safe and efficient treatment in elderly patients with essential thrombocythemia. Leukemia 21, 2071-2072 (2007).

40. Kuriakose, E. T. et al. JAK2V617F allele burden is reduced by busulfan therapy: a new observation using an old drug. Haematologica 98, e135-e137 (2013).
41. Alvarez-Larran, A. et al. Busulfan in patients with polycythemia vera or essentia thrombocythemia refractory or intolerant to hydroxyurea. Ann. Hematol. 93 2037-2043 (2014)

42. Begna, K. et al. Busulfan for the treatment of myeloproliferative neoplasms: the Mayo Clinic experience. Blood Cancer J. 6, e427 (2016).

43. Douglas, G. et al. Busulfan is effective second-line therapy for older patients with Philadelphia-negative myeloproliferative neoplasms intolerant of or unresponsive to hydroxyurea. Leuk. Lymphoma 58, 89-95 (2017).

44. Tefferi, A. et al. Survival and prognosis among 1545 patients with contemporary polycythemia vera: an international study. Leukemia $\mathbf{2 7}, 1874-1881$ (2013).

45. Brodsky, I. Busulfan versus hydroxyurea in the treatment of polycythemia vera (PV) and essential thrombocythemia (ET). Am. J. Clin. Oncol. 21, 105-106 (1998).

46. Mela Osorio, M. J. et al. Long-term follow-up of essential thrombocythemia patients treated with anagrelide: subgroup analysis according to JAK2/CALR MPL mutational status. Eur. J. Haematol. 96, 435-442 (2016).

47. Storen, E. C. \& Tefferi, A. Long-term use of anagrelide in young patients with essential thrombocythemia. Blood 97, 863-866 (2001).

48. Jurgens, D. J., Moreno-Aspitia, A. \& Tefferi, A. Anagrelide-associated cardiomyopathy in polycythemia vera and essential thrombocythemia. Haematologica 89, 1394-1395 (2004).

49. Mazzucconi, M. G. et al. A long-term study of young patients with essentia thrombocythemia treated with anagrelide. Haematologica 89, 1306-1313 (2004).

50. Engel, P. J., Johnson, H., Baughman, R. P. \& Richards, A. I. High-output heart failure associated with anagrelide therapy for essential thrombocytosis. Ann. Intern. Med. 143, 311-313 (2005).

51. Passamonti, F. et al. Long-term follow-up of young patients with essentia thrombocythemia treated with pipobroman. Ann. Hematol. 83, 495-497 (2004).

52. Mazzucconi, M. G. et al. Pipobroman therapy of essential thrombocythemia Scand. J. Haematol. 37, 306-309 (1986).

53. De Sanctis, $V$. et al. Long-term evaluation of 164 patients with essentia thrombocythaemia treated with pipobroman: occurrence of leukaemic evolution. Br. J. Haematol. 123, 517-521 (2003).

54. Kiladjian, J. J., Chevret, S., Dosquet, C., Chomienne, C. \& Rain, J. D. Treatment of polycythemia vera with hydroxyurea and pipobroman: final results of a randomized trial initiated in 1980. J. Clin. Oncol. 29, 3907-3913 (2011).

55. Tefferi, A. \& Passamonti, F. Essential thrombocythemia and pregnancy: Observations from recent studies and management recommendations. Am. J. Hematol. 84, 629-630 (2009).

56. Gangat, N., Wolanskyj, A. P., Schwager, S. \& Tefferi, A. Predictors of pregnancy outcome in essential thrombocythemia: a single institution study of 63 pregnancies. Eur. J. Haematol. 82, 350-353 (2009).

57. Beauverd, Y. et al. Pegylated interferon alpha-2a for essential thrombocythemia during pregnancy: outcome and safety. A case series. Haematologica 101, e182-e184 (2016)

58. Valera, M. C., Parant, O., Vayssiere, C., Arnal, J. F. \& Payrastre, B. Essential thrombocythemia and pregnancy. Eur. J. Obstet. Gynecol. Reprod. Biol. 158, 141-147 (2011). 